\title{
Functional Capillary Density for in Vivo Estimation of Intestinal Perfusion using Real-Time Confocal Endomicroscopy
}

Luigi Schiraldi ${ }^{1}$, Francesco Marchegiani ${ }^{1}$, Michele Diana ${ }^{1,2 *}$, Véronique Lindner ${ }^{3}$, Eric NolI ${ }^{4}$, Pierre Diemunsch ${ }^{4}$ and Jacques Marescaux ${ }^{1,2}$

${ }^{1} I R C A D$, Research Institute Against Cancer of the Digestive System, Strasbourg, France

${ }^{2} I \mathrm{HU}$, Institute for Minimally Invasive Image-Guided Therapies, Strasbourg, France

${ }^{3}$ Department of Pathology, University Hospital of Strasbourg, France

${ }^{4}$ Department of Anaesthesiology, University Hospital of Strasbourg, France

\begin{abstract}
Background and aim: Confocal Laser Endomicroscopy (CLE) has been successfully used to appreciate microcirculation changes of the digestive mucosa. Our aim was to evaluate CLE scanning complemented by functional capillary density area (FCD-A) estimation to define the micro-vessel status in a reiterate, long-lasting porcine model of bowel ischemia.
\end{abstract}

Materials and methods: A laparotomy was performed in 4 pigs, and a segmental $(3-4 \mathrm{~cm})$ ischemia of the sigmoid colon was induced with vascular clamps. Ischemic and perfused regions were clinically defined. After an injection of $5 \mathrm{ml}$ of sodium fluorescein $10 \%$ (Fluocyne, SERB, Paris, France), the Cellvizio ${ }^{\mathrm{m}}$ confocal probe (Mauna $^{\circ}$ Kea Technologies, Paris, France) was directly applied onto the mucosa's surface through a full-thickness enterotomy. Both ischemic area (IA) and control region-perfused area (PA) - were scanned and video sequences were recorded.

Results: Confocal evaluation of the ischemic area revealed a different aspect of the mucosal tissue when compared to the normal perfused area. Statistically, FCD-A at the perfused area was significantly higher when compared to the ischemic area, irrespective of the time point. After 1 hour, FCD-A was $(0.189 \pm 0.094$ vs. $0.365 \pm$ $0.030 ; p=0.0001)$, after 2 hours $(0.252 \pm 0.056$ vs. $0.389 \pm 0.024 ; p<0.0001)$, after 3 hours $(0.252 \pm 0.050$ vs. 0.353 $\pm 0.030 ; p=0.0001)$ and after 4 hours $(0.262 \pm 0.044$ vs. $0.358 \pm 0.019 ; p<0.0001)$, at ischemic and perfused areas respectively.

Conclusions: Confocal imaging allows real-time discrimination between perfused and ischemic areas of the bowel using morphological clues, while the functional capillary density area adds a quantitative measurement.

Keywords: Confocal laser endomicroscopy; Functional capillary density area; Cellvizio" system; Quantitative assessment of bowel perfusion

\section{Introduction}

Confocal laser endomicroscopy (CLE) is a high-resolution imaging modality allowing for real-time in vivo virtual biopsies with in vivo magnification up to $1000 \mathrm{X}$ [1] and spatial lateral resolution ranging from 1 to $3.5 \mu \mathrm{m}[2]$.

CLE systems include through-the-scope probes (probe-based CLE, or pCLE), e.g. the Cellvizio"' system (Mauna Kea Technologies, Paris, France) (Figure 1) or endoscope-based CLE (eCLE), e.g. the Pentax Confocal Endomicroscope (EC-3870CIFK, Pentax, Tokyo, Japan) [2]. The main advantages of eCLE when compared to pCLE are the larger visual field $(475 \times 475 \mu \mathrm{m}$ vs. $240-325 \times 240-325)$, and the adjustable imaging plane depth [1]. These features improve the performance of eCLE in terms of accuracy [1]. However, pCLE is more versatile since various sized probes can be inserted through the operative channel of standard endoscopes, as well as through biopsy needles (needle-based CLE). Additionally, pCLE allows to perform a virtual biopsy under visual control [2].

Image acquisition in CLE requires a fluorophore, which can be administered using a systemic injection (mainly sodic fluorescein) or through a topic application (acriflavin or violet cresyl). Those "contrast" agents may add some dynamic and functional data to morphological information, making the confocal method more than a mere digital version of histopathology.

Current clinical applications for pCLE in GI endoscopy include postresection follow-up of colonic lesions [3], diagnosis of indeterminate biliary strictures [4], Barrett's esophagus surveillance, and posttreatment pathologic assessment $[5,6]$. Evolving applications include inflammatory bowel disease [7], gastric diseases [8], differentiation of colorectal polyps [9], and pancreatic cysts [10]. In the experimental setting, CLE can provide in vivo analysis of microcirculation [11,12]. Recently, CLE was used to appreciate microcirculation changes of the digestive mucosa during septic shock in the experimental and clinical setting [13]. Our group demonstrated the ability of the Cellvizio system to accurately discriminate between perfused and ischemic areas in a 1-hour model of bowel ischemia [14]. The aim of this experimental study was to evaluate confocal scanning complemented by functional capillary density area (FCD-A) estimation to define the micro-vessel status in a reiterate, long-lasting porcine model of bowel ischemia.

\section{Materials and Methods}

\section{Animals}

Four female pigs (Sus scrofa domesticus, ssp. Large White), mean

*Corresponding author: Michele Diana, 1, place de l'Hôpital, 67091 Strasbourg, France, Tel: +33 651186 529; Fax: +33 388119 099; E-mail: michele.diana@ircad.fr

Received May 22, 2015; Accepted June 22, 2015; Published June 24, 2015.

Citation: Schiraldi L, Marchegiani F, Diana M, Lindner V, Noll E, et al. (2015) Functional Capillary Density for in Vivo Estimation of Intestinal Perfusion using Real-Time Confocal Endomicroscopy. J Cytol Histol 6: 334. doi:10.4172/21577099.1000334

Copyright: (C) 2015 Schiraldi L, et al. This is an open-access article distributed under the terms of the Creative Commons Attribution License, which permits unrestricted use, distribution, and reproduction in any medium, provided the original author and source are credited. 
Citation: Schiraldi L, Marchegiani F, Diana M, Lindner V, Noll E, et al. (2015) Functional Capillary Density for in Vivo Estimation of Intestinal Perfusion using Real-Time Confocal Endomicroscopy. J Cytol Histol 6: 334. doi:10.4172/2157-7099.1000334

weight of $31.025 \pm 3.34 \mathrm{~kg}$, and were used in this non-survival study. The protocol (No. 38.2012.01.039) was approved by the local Ethical Committee on Animal Experimentation. Animals were managed according to ARRIVE guidelines [15] and in accordance with French laws for animal use and care and according to the directives of the European Community Council (2010/63/EU). Twenty-four hours before surgery, pigs were fasted with free access to water. Intramuscular injection of ketamine $(20 \mathrm{mg} / \mathrm{Kg})$ and azaperone $(2 \mathrm{mg} / \mathrm{Kg})$ (Stressnil, Janssen-Cilag, Belgium) were administered 10 minutes before surgery. Induction was achieved using intravenous propofol $(3 \mathrm{mg} / \mathrm{Kg})$ combined with rocuronium $(0.8 \mathrm{mg} / \mathrm{Kg})$. Anesthesia was maintained with $2 \%$ isoflurane. At the end of the procedure, animals were sacrificed with an intravenous injection of a lethal dose of potassium chloride.

\section{Surgical procedure and Confocal Endomicroscopy assessment}

A median laparotomy was performed in all pigs, and segmental $(3-4 \mathrm{~cm})$ ischemia of the sigmoid colon was induced by occluding terminal arterial branches with vascular clamps. Ischemic regions were clinically defined through observation and taking into account the vascular anatomy. After an injection of $5 \mathrm{~mL}$ of sodium fluorescein 10\% (Fluocyne, SERB, Paris, France), the Cellvizio" pCLE Gastroflex UHD was used to perform a scan of the sigmoid mucosa (Figure 1). The probe was directly applied onto the mucosa's surface through a fullthickness enterotomy. Confocal scanning was performed at 12 frames per second as previously described [16].

Both the ischemic area (IA) and the control region-perfused area (PA) - were scanned, and video sequences were recorded. A mean of $656 \pm 360$ and $408 \pm 200$ frames were examined for IA and PA respectively. Confocal scanning was reiterated every hour, for 4 hours of ischemia. Scanning included 48 ischemic areas and 48 perfused areas analyzed at different time points.

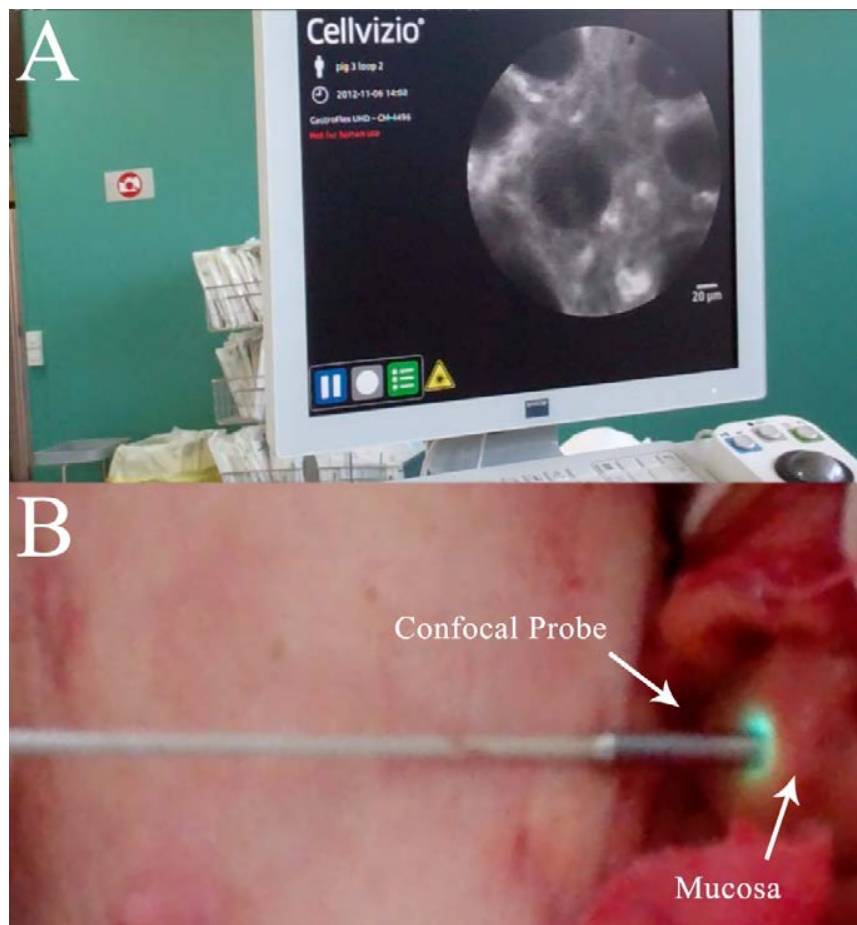

Figure 1: Probe-based Confocal Laser Endomicroscopy (pCLE). A) Cellvizio monitor showing perfused sigmoid mucosa. B) The Cellvizio ${ }^{\text {Tw }}$ pCLE Gastroflex UHD was applied directly onto the sigmoid mucosa through full-thickness enterotomies.

\section{Functional Capillary Density Area (FCD-A) assessment}

Ninety-six relevant, non-redundant images were analyzed using the IC Viewer software (version 3.8.5) (Mauna Kea Technologies, Paris, France). Mean capillary diameter, total capillary area per field, and Functional Capillary Density Area (FCD-A) were computed. The vessel detection tool explores diameters ranging from half the diameter of interest (DOI) to twice the DOI (as declared by the manufacturer). In order to obtain the FCD-A index, the mean capillary diameter is multiplied by the total vessel length, and the result is then divided by the entire round image area (Figure 2). The automatic vessel detection is based on the presence of fluorescence contrast in elongated shapes [13]. In order to prevent erroneous capillary detection due to a possible fluorescein leakage inside the lumen of mucosal crypts, the circularshaped area within the crypts was subtracted to the region of interest (Figure 3).

\section{Histology}

Full-thickness biopsies were sampled immediately after confocal scanning at both ischemic and perfused areas and fixed in $4 \%$ of buffered formalin. Paraffin-embedded sections ( $4 \mu \mathrm{m}$ thick) were stained with hematoxylin and eosin. Six sections per biopsy were analyzed. A semiquantitative ischemia score was applied by a pathologist to normal and ischemic areas. The score scale was as follows: $0=$ normal mucosa; $1=$ partial epithelial edema and necrosis; $2=$ diffuse swelling and necrosis of epithelium; $3=$ necrosis with submucosal neutrophil infiltration; $4=$ widespread necrosis and massive neutrophil infiltration and hemorrhage.

\section{Statistics}

Statistics were performed using the GraphPad Prism software. A Student's t-test was performed to calculate differences between ischemic and perfused areas. Differences were considered statistically significant for $p$ values $<0.01$.

\section{Results}

Confocal evaluation of the ischemic area revealed a different aspect of the mucosal tissue when compared to the normal perfused area. CLE identifies the ischemic area with blurred images and swelling with an increased demarcation of the cryptal border, due to increased basolateral permeability. The presence of "target cells" is also characteristic, defined by the presence of a hyper-fluorescent crypt centre and by the distortion of the enterocyte silhouette. These typical features cannot be found in the normally perfused area (Figure 4 and 5).

Statistically, FCD-A at the perfused area was significantly higher when compared to the ischemic area, irrespective of the time point. After 1 hour, FCD-A was $(0.189 \pm 0.094$ vs. $0.365 \pm 0.030$; $\mathrm{p}=0.0001)$, after 2 hours $(0.252 \pm 0.056$ vs. $0.389 \pm 0.024 ; \mathrm{p}<0.0001)$, after 3 hours $(0.252 \pm 0.050$ vs. $0.353 \pm 0.030 ; \mathrm{p}=0.0001)$, and after 4 hours $(0.262$ \pm 0.044 vs. $0.358 \pm 0.019 ; \mathrm{p}<0.0001)$, at ischemic and perfused areas respectively.

Overall, the ischemia score was low, ranging from 0 to 2 . The CLEbased score of the ischemic area was significantly higher than the one assessed with a standard pathology $(1.71 \pm 0.49$ vs. $1 \pm 0$; $\mathrm{p}=0.0082)$ The perfused area was invariably normal at standard histology (score 0 ) and at confocal evaluation, except in one case.

\section{Discussion}

CLE is an emerging real-time diagnostic tool, which can 
Ischemic Area (IA)

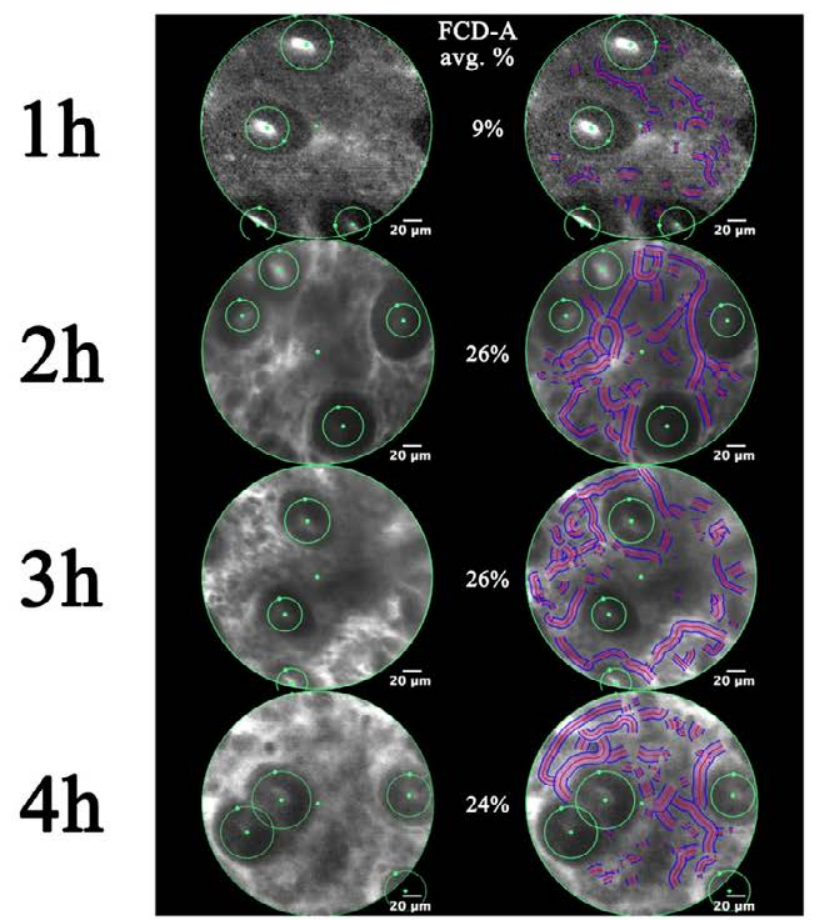

Perfused Area (PA)

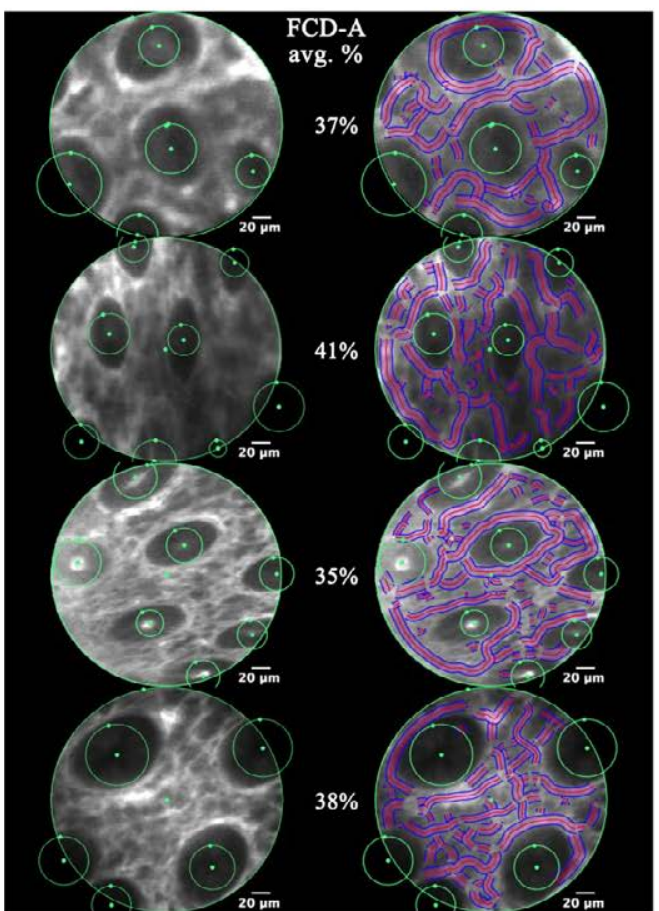

Figure 2: Functional Capillary Density Area (FCD-A) assessment. FCD-A assessed with the IC Viewer software (version 3.8.5). The FCD-A could precisely discriminate between ischemic and perfused areas at any time point. The green circles were applied to both ischemic and perfused areas to subtract fluorescein leaks occurring in the middle of the crypts.

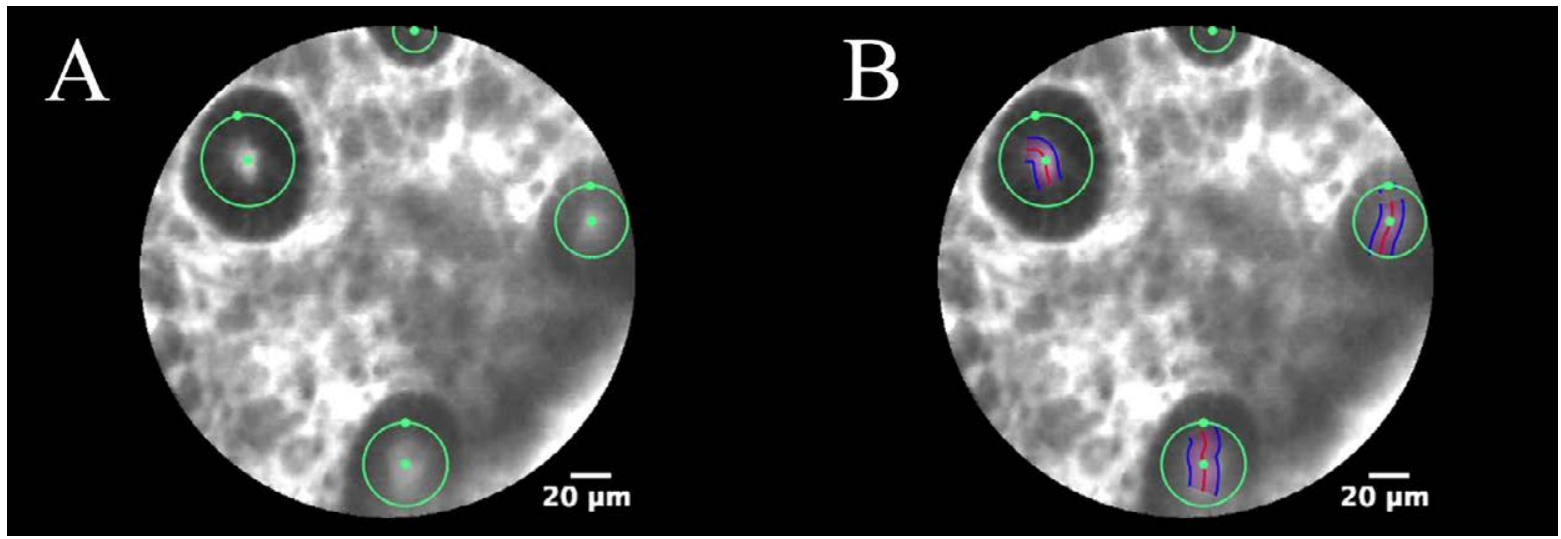

Figure 3: Post-processing of vessel detection. A) Green circles outline areas to be subtracted since B) there was erroneous vessel detection within the intestinal crypts. The erroneous detection of vessels inside the lumen was prevented by post-processing images and excluding circular areas around and within the crypts.

complement an endoscopic assessment by performing a digital biopsy. It is being progressively implemented in the management of neoplastic or inflammatory diseases including gastrointestinal, urinary or respiratory tract [2] diseases. In addition, CLE can provide information about the microcirculatory status of tissues [12].

More particularly, CLE might provide a quantitative real-time assessment of bowel perfusion, which could have a relevant impact on various clinical situations, including the management of intensive care unit patients or the intraoperative evaluation of stoma or bowel stump perfusion.
Yasumura et al. used a charge-coupled microscopic device to analyze perfusion from the serosal layer in a bowel ischemia model. Authors could calculate a ratio of circulating blood cells to the functional bowel vascular bed as a predictive index of intestinal survival [17]. This type of accurate quantification of intestinal perfusion, when it is not time-consuming or overwhelmingly expensive, might represent a paradigmatic shift towards micro-image-guided therapies, allowing for a Doppler-like real-time examination at a microscopic scale.

Schmidt et al. were able to describe sepsis-related changes in mucosal microcirculation in a porcine model of septic shock and also 
Citation: Schiraldi L, Marchegiani F, Diana M, Lindner V, Noll E, et al. (2015) Functional Capillary Density for in Vivo Estimation of Intestinal Perfusion using Real-Time Confocal Endomicroscopy. J Cytol Histol 6: 334. doi:10.4172/2157-7099.1000334
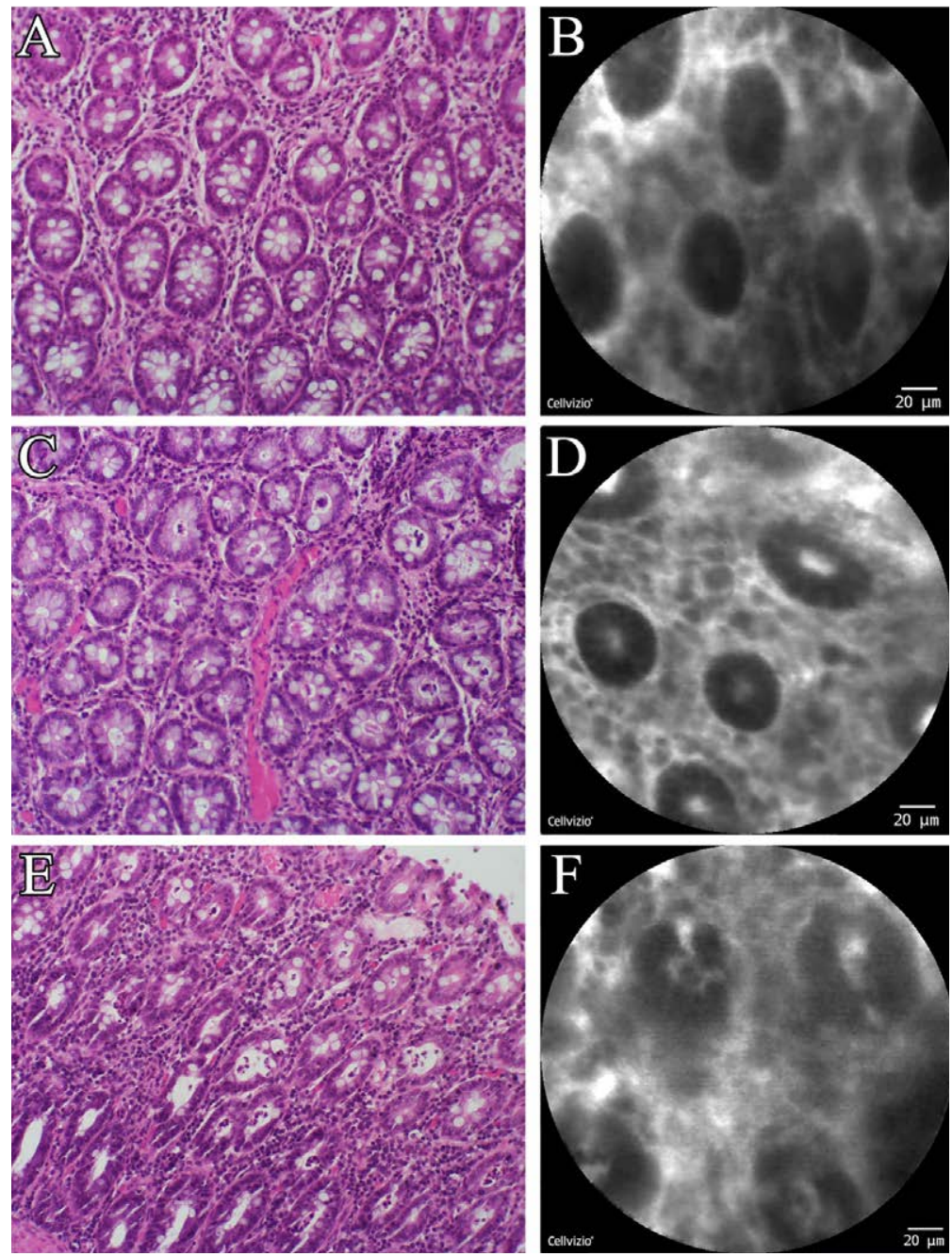

Figure 4: Comparative images: standard histology versus confocal analysis. A blinded pathologist assigned an ischemia score to histological and confocal images. A) 20X Hematoxylin-eosin (HE), and B) confocal imaging score 0 (normal mucosa), C) 20X HE, and D) confocal imaging score 1 (submucosal congestion and swelling), E) 20X HE, and F) confocal imaging score 2 (diffuse swelling and necrosis of epithelium).

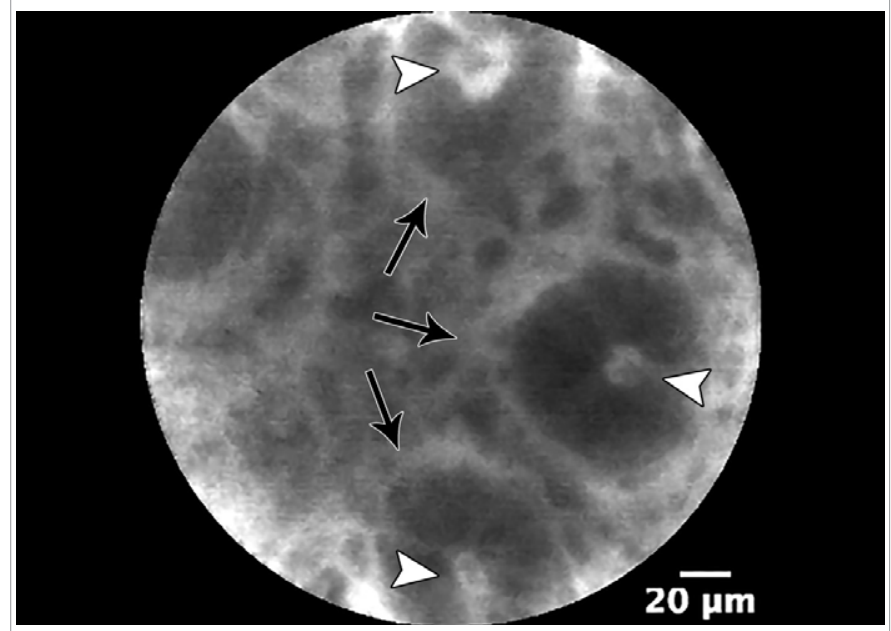

Figure 5: Confocal description of ischemic area. Black arrows indicate an enhanced cryptal border in the colonic mucosa. White arrowheads show the fluorescein leakage inside the cryptal lumen. in patients using the Cellvizio" system and a computer-based evaluation of functional capillary density area (FCD-A). In the future, such detectable changes could potentially help to adjust fluid resuscitation regimens, given the complexity of fluid management in critical patients presenting a shock of abdominal origin [13].

In a porcine laparoscopic model of bowel ischemia limited to 1 hour, we could observe that a real-time confocal morphological evaluation provides early and specific clues to identify the vascular status. CLE was more specifically used to confirm and validate the accuracy of a surgical navigation system to detect bowel perfusion, based on the dynamics of the fluorescent signal [18-20].

The aim was to perform assessments over time and to complement the morphological analysis with FCD-A computation. For that purpose, we designed the current open surgery and long-lasting model of sigmoid ischemia (up to 4 hours).

The vessel detection tool, which is included in the software provided by Mauna Kea Technologies (IC-Viewer 3.8.5), may provide the FCD-A index, which can estimate vascular flow. The FCD-A index is based on fluorescein signal detection. In healthy mucosa, fluorescein is confined 
Citation: Schiraldi L, Marchegiani F, Diana M, Lindner V, Noll E, et al. (2015) Functional Capillary Density for in Vivo Estimation of Intestinal Perfusion using Real-Time Confocal Endomicroscopy. J Cytol Histol 6: 334. doi:10.4172/2157-7099.1000334

to the vessels' lumen. Ischemic injury produces leaks of fluorescein by increasing vascular and basolateral intestinal permeability. This fluorescent leakage is found primarily in the cryptal lumen, as an early indirect sign of ischemia. This creates the aspect of "target cells" and subsequently forms a "palisade" aspect due to an increased spacing of the cryptal border. A prolonged ischemic injury leads to a pooling phenomenon in homogeneously distributed on the mucosal surface, creating visible artefacts during the software vessel detection process. In order to optimize vessel detection, a post-processing analysis is required to subtract fluorescein leaking areas around and within the intestinal crypts.

When comparing the ischemia score applied by a blinded pathologist (VL) to both techniques, it was clear that the morphological confocal analysis tends to overestimate injuries when compared to standard histology. However, when it comes to a standard pathology, the perfused area was consistently deemed to be strictly normal and only mild damage was found at the ischemic areas, irrespective of ischemic time. Such findings were different from those made with CLE. Findings made with CLE demonstrated more discriminant signs of ischemic injury, which matched the FCD-A analysis. This study model was probably limited by the short ischemic segment, in which some reperfusion might have occurred, protecting the mucosa from further degeneration.

\section{Conclusions}

Confocal imaging allows for real-time discrimination between the bowel's perfused and ischemic areas using morphological clues, while the functional capillary density area adds a quantitative measurement. This micro-image quantitative analysis might be helpful in clinical conditions requiring an accurate assessment of bowel perfusion, such as in the presence of a stoma or in the management of a shock of abdominal origin.

\section{Acknowledgments}

Authors are grateful to Mathilde Raux-Defossez, Christopher Burel, and Guy Temporal, for their valuable assistance in proofreading the manuscript.

\section{References}

1. Buchner AM, Wallace MB (2015) In-vivo microscopy in the diagnosis of intestinal neoplasia and inflammatory conditions. Histopathology 66: 137-146.

2. ASGE Technology Committee (2014) Confocal laser endomicroscopy. Gastrointest Endosc 80: 928-938.

3. Shahid MW, Buchner AM, Coron E, Woodward TA, Raimondo M, et al (2012) Diagnostic accuracy of probe-based confocal laser endomicroscopy in detecting residual colorectal neoplasia after EMR: a prospective study. Gastrointest Endosc 75: 525-533.

4. Caillol F, Filoche B, Gaidhane M, Kahaleh M (2013) Refined probe-based confocal laser endomicroscopy classification for biliary strictures: the Paris Classification. Dig Dis Sci 58: 1784-1789.

5. Leung KK, Maru D, Abraham S, Hofstetter WL, Mehran R, et al. (2009) Optical EMR: confocal endomicroscopy-targeted EMR of focal high-grade dysplasia in Barrett's esophagus. Gastrointest Endosc 69: 170-172.

6. Sharma P, Bansal A (2006) Toward better imaging of Barrett's esophagus--see more, biopsy less! Gastrointest Endosc 64: 188-192.

7. Neumann H, Vieth M, Atreya R, Neurath MF, Mudter J (2011) Prospective evaluation of the learning curve of confocal laser endomicroscopy in patients with IBD. Histol Histopathol 26: 867-872.

8. Zhang JN, Li YQ, Zhao YA, Yu T, Zhang JP, et al. (2008) Classification of gastric pit patterns by confocal endomicroscopy. Gastrointest Endosc 67: 843-853.

9. Kiesslich R, Burg J, Vieth M, Gnaendiger J, Enders M, et al. (2004) Confocal laser endoscopy for diagnosing intraepithelial neoplasias and colorectal cancer in vivo. Gastroenterology 127: 706-713

10. Konda VJ, Meining A, Jamil LH, Giovannini M, Hwang JH, et al. (2013) A pilot study of in vivo identification of pancreatic cystic neoplasms with needle-based confocal laser endomicroscopy under endosonographic guidance. Endoscopy 45: 1006-1013.

11. Gruber S, Spielauer I, Böhme S, Baron D, Markstaller K et al. (2015) Real-time in-vivo imaging of pulmonary capillary perfusion using probe-based confocal laser scanning endomicroscopy in pigs: An interventional laboratory study. Eur J Anaesthesiol 32: 392-399.

12. Laemmel E, Genet M, Le Goualher G, Perchant A, Le Gargasson JF, et al. (2004) Fibered confocal fluorescence microscopy (Cell-viZio) facilitates extended imaging in the field of microcirculation. A comparison with intravital microscopy. Journal of vascular research $4: 400-411$.

13. Schmidt C, Lautenschläger C, Petzold B, Sakr Y, Marx G, et al. (2013) Confocal laser endomicroscopy reliably detects sepsis-related and treatment-associated changes in intestinal mucosal microcirculation. $\mathrm{Br}$ J Anaesth 11: 996-1003.

14. Diana M, Dallemagne B, Chung H, Nagao $Y$, Halvax $P$, et al. (2014) Probebased confocal laser endomicroscopy and fluorescence-based enhanced reality for real-time assessment of intestinal microcirculation in a porcine mode of sigmoid ischemia. Surg Endosc 28: 3224-3233.

15. Kilkenny C, Browne WJ, Cuthill IC, Emerson M, Altman DG (2010) Improving bioscience research reporting: the ARRIVE guidelines for reporting animal research. PLoS Biol 8: e1000412.

16. Fitoussi V, Faye N, Chamming's F, Clement O, Cuenod CA, et al. (2013) In vivo imaging of tumor angiogenesis using fluorescence confocal videomicroscopy. $J$ Vis Exp.

17. Yasumura M, Mori Y, Takagi H, Yamada T, Sakamoto K, et al. (2003) Experimental model to estimate intestinal viability using charge-coupled device microscopy. Br J Surg 90: 460-465

18. Diana M, Agnus V, Halvax P, Liu YY, Dallemagne B, et al. (2015) Intraoperative fluorescence-based enhanced reality laparoscopic real-time imaging to assess bowel perfusion at the anastomotic site in an experimental model. $\mathrm{Br} \mathrm{J}$ Surg 102: e169-e176.

19. Diana M, Halvax P, Dallemagne B, Nagao Y, Diemunsch P, et al. (2014) Realtime navigation by fluorescence-based enhanced reality for precise estimation of future anastomotic site in digestive surgery. Surg Endosc 28: 3108-3118.

20. Diana M, Noll E, Diemunsch P, Dallemagne B, Benahmed MA, et al. (2014) Enhanced-reality video fluorescence: a real-time assessment of intestinal viability. Ann Surg 259: 700-707. 